\title{
Azure dyes as new photosensitizer prototypes to application in photodynamic therapy against Candida spp.
}

\author{
Leonardo M. Moreira $^{\text {a }}$, Juliana P. Lyon ${ }^{\mathrm{b}}$, Suellen M.S. Tursi ${ }^{\mathrm{c}}$, Isis Trajano ${ }^{\mathrm{c}}$, \\ Monalisa P. Felipe ${ }^{c}$, Maricília S. Costa ${ }^{c}$, Máira R. Rodrigues ${ }^{d}$, Lúcia Codognoto $^{\mathrm{e}}$ and \\ Hueder P.M. de Oliveira ${ }^{\mathrm{e}, *}$ \\ ${ }^{a}$ Departamento de Engenharia de Biossistemas, Universidade Federal de São João Del Rei, São João \\ del Rei, MG, Brazil \\ ${ }^{\mathrm{b}}$ Departamento de Ciências Naturais, Universidade Federal de São João Del Rei, São João del Rei, \\ $M G$, Brazil \\ ${ }^{\mathrm{c}}$ Instituto de Pesquisa e Desenvolvimento, Universidade do Vale do Paraíba, São José dos Campos, SP, \\ Brazil \\ ${ }^{\mathrm{d}}$ Departamento de Ciência e Tecnologia, Universidade Federal Fluminense, Polo Universitário de Rio \\ das Ostras, Rio das Ostras, RJ, Brazil \\ e Universidade Camilo Castelo Branco, São José dos Campos, SP, Brazil
}

\begin{abstract}
Infections caused by Candida albicans are of increasing concern, especially considering immunodepressed patients. The toxicity of most antifungal agents, the great number of cases with recidives, as well as the emergence of resistant samples has provoked the evaluation of new forms of therapy. In this context, the photodynamic therapy (PDT) presents auspicious antimicrobial properties, stimulating the development of trials employing several kinds of photosensitizers. In the present work, the application of different kind of Azure dyes as photosensitizer in PDT against $C$. albicans was evaluated through instrumental measurements of electronic spectroscopy. In fact, the values of optical density were a precise indicator of the growth inhibition of the microorganisms. Indeed, Azures are phenothiazinium derivatives that constitute a very relevant class of compounds with several biomedical applications, such as photoantimicrobial therapy against local bacterial infection, tuberculosis, trypanosomiasis, malaria, Rickettsia, yeasts, viral infection $n$ and cancer. Azure A, Azure B, Azure A thiocyanate, Azure B BF 4 , Azure A eosinate are the dyes tested against $C$. albicans. The results denoted completely distinct behaviors to the different types of Azure compound evaluated in this work. In fact, Azure A and Azure A eosinate presented significant results when irradiated with $56 \mathrm{~J} / \mathrm{cm}^{2}$, since the growth inhibition of $C$. albicans reached approximately $60 \%$. This Azure compounds have significant potential to be employed as photosensitizer (PS) in PDT, especially in cases of mucocutaneous candidosis. The spectroscopic evaluation was very effective to the detection of slight alterations in the growth of the microorganisms, denoting that this kind of analysis is an excellent alternative to determine growth inhibition of Candida albicans. The experimental data are discussed in details in agreement with recent results from literature.
\end{abstract}

Keywords: Photodynamic therapy (PDT), photosensitizer (PS), Azures, Candida albicans

\footnotetext{
${ }^{*}$ Corresponding author: Hueder P.M. de Oliveira, Universidade Camilo Castelo Branco/Rod. Presidente Dutra Km 139 Eugênio de Melo, São José dos Campos, SP 12247-004, Brazil. Tel./Fax: +55 1239054648 - R 209; E-mails: huederpaulo@ yahoo.com.br, leonardomarmo@gmail.com.
} 


\section{Introduction}

C. albicans is commensal yeast present in about $20 \%$ of healthy individuals [10]. However, some factors are predisposing for the development of candidosis, such as immunodeficiencies, malignant diseases, radiotherapy, long-term treatment with antibacterial drugs and age extremes [13]. Moreover, both cutaneous and mucocutaneous candidosis may appear in healthy individuals with local predisposing conditions, such as denture wearing, moist wrinkles in skin and altered local $\mathrm{pH}$ [14].

Amphotericin B and azolics, such as ketoconazole, fluconazole and itraconazole, have been considered efficient in the treatment of both systemic and local candidosis. However, the prophylactic use of azolic drugs in immunodepressed patients led to the selection of resistant strains [16]. Besides, the increase in the number of infections caused by Candida species other than C. albicans also contributed to the emergence of isolates that are resistant to amphotericin B and fluconazole [17]. It is also important to notice that the treatment with antifungal drugs may be prolonged and recidives are very common.

Photodynamic therapy (PDT) is a promising therapeutic treatment for various tumors and nonmalignant diseases. The treatment requires a dye and a light source. The light activates a dye deposited on the target organism and sensitizes it, transforming the oxygen at molecular level into reactive oxygen species (ROS), such as singlet oxygen, which is cytotoxic to microorganisms $[2,6,8,9,24]$. Photodynamic therapy has been originally developed for the treatment of malignant diseases. However, other applications have emerged. Photodynamic antimicrobial chemotherapy (PACT) is an alternative modality of treatment for microbial infections. The therapy consists in applying a photosensitizer and a light source, causing selective microbial death [11]. PACT has been successfully employed against leishmaniasis [1, 4], trichomoniasis [18], as well as in the destruction of gram-positive and gram-negative bacteria [22, 24]. PACT has been also tested against yeasts and dermatophytes $[3,15]$.

In this context, the development of new or coadjutants treatments for superficial candidosis has a great relevance. It is important to notice that infections produced by $C$. albicans, mainly those that occur in immunodepressed patients, constitute a focus of interest to several groups. In fact, the development of new treatments is a point of great relevance due to the appearance of resistant biological samples. Several works have evaluated the use of photosensitizers and light to kill yeasts and molds [7]. However, there are few studies regarding the physicochemical properties necessary in a photosensitizer to be effective in photodynamic antifungal chemotherapy.

The present work evaluated the employment of Azures (Azure A, Azure B, Azure A thiocyanate, Azure B BF 4 and Azure A eosinate, see Fig. 1) as photosensitizers to be employed in PDT against C. albicans. Azures are phenothiazinium derivatives that constitute a very relevant class of compounds with biomedical applications. The phenothiazinium dyes are known to localize in the plasma membrane of yeasts. Consequently, this is the cellular structure damaged upon illumination and it has been proposed that the increased permeability resulting from such damage is the reason for cell death [7]. These compounds have also been recently employed in a great number of studies with biological and chemical relevance, such as development of new analytical methodologies aiming to detect compounds of envi-

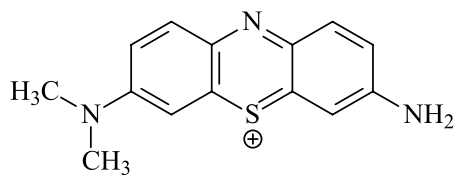

Azure A<smiles></smiles>

Azure B

Fig. 1. Structures of Azures A and B. 
ronmental importance [12] as well as evaluations focused on the biochemical mechanisms of Alzheimer disease [20]. Phenothiazinium salts offer more scope in terms of the therapy of disease states than other dye types, including that employed as photosensitizers (PS). Thus, dyes as methylene blue and toluidine blue $\mathrm{O}$ have been lead compounds in drug research against local bacterial infection, tuberculosis, trypanosomiasis, malaria, Rickettsia illness, yeast infection, viral blood colonization and cancer [23]. In this work, instrumental measurements of optical density were employed in order to determine the level of growth inhibition of $C$. albicans. Indeed, the electronic absorbance in the spectral region of visible was quite able to identify modifications in the presence of the microorganism. The results obtained in the present article indicate that these compounds present significant potential to be used as photosensitizer agents in PDT. The data are evaluated and discussed in details in agreement with recent reports from literature.

\section{Materials and methods}

Initially, a standardized suspension $\left(10^{6}\right.$ viable cells $\left.\mathrm{ml}^{-1}\right)$ of $C$. albicans ATCC 10231 was prepared as described in the literature [19]. A Candida suspension $\left((1-5) \times 10^{5}\right.$ cells $\left.\mathrm{ml}^{-1}\right)$ was seeded and incubated in the dark for $5 \mathrm{~min}$ at room temperature in the presence of different concentrations of phenothiazinium derivatives Azure A, Azure B, Azure A thiocyanate, Azure $\mathrm{B} \mathrm{BF}_{4}{ }^{-}$and Azure A eosinate, ranging from 0.01 to $0.5 \mathrm{mg} \cdot \mathrm{ml}^{-1}$, in a final volume of $0.2 \mathrm{ml}$. Cells incubated in sterile physiological solution alone were included as a control. After this period, the covers of the 96-well plates were removed, and the plates were illuminated with the appropriate light at room temperature, according to the method described by Souza et al. [19]. The light source used was a diode laser InGaAlP (Photon Laser, DMC, São Carlos, Brazil), with output power of $35 \mathrm{~mW}$ and wavelength of $684 \mathrm{~nm}$. The energy dose was of 28 or $56 \mathrm{~J} / \mathrm{cm}^{2}$, varying the time of irradiation. Aliquots of $50 \mu \mathrm{l}$ were taken before and after illumination so that we could determine both the number of colony forming units (CFUs). The contents of the wells were properly homogenized before being sampled. To determine the number of CFUs, we diluted aliquots 1000-fold in sterile physiological solution and spread them evenly on a Petri dish containing Sabouraud dextrose agar medium. The colonies were incubated at $37^{\circ} \mathrm{C}$ for $48 \mathrm{~h}$, and the number of colony forming units per milliliter (CFUs ml${ }^{-1}$ ) was determined. The experiments were performed in the dark and under aseptic conditions. In the spectroscopic analysis, it was evaluated the optical density at $570 \mathrm{~nm}$, which is an interesting wavelength as function of the absorption of the several species of aggregates, such as dimmers, trimers and oligomers that present wavelength of maximum absorption blue-shifted in relation to the monomeric form. In this way, even with significant aggregation, a representative excitation of the photosensitizers would occur, favoring a higher photodynamic action. Furthermore, in this wavelength $(570 \mathrm{~nm})$, the absorption of dyes inherent to the biological medium, such as hemoglobin and melanin, is significantly low, implying that the competition by the photons in the excitation process between Azures and biological dyes would be decreased. For consequence, a suitable excitation would be obtained independently of the level of aggregation of the Azures. All the experiments were made in triplicate, being that it was considered the medium value between the three analysis.

\section{Results and discussion}

Figure 2 presents the results regarding the effect of Azure B on Candida yeasts. It is possible to note that Azure B does not present an effective inhibition of $C$. albicans growth. 


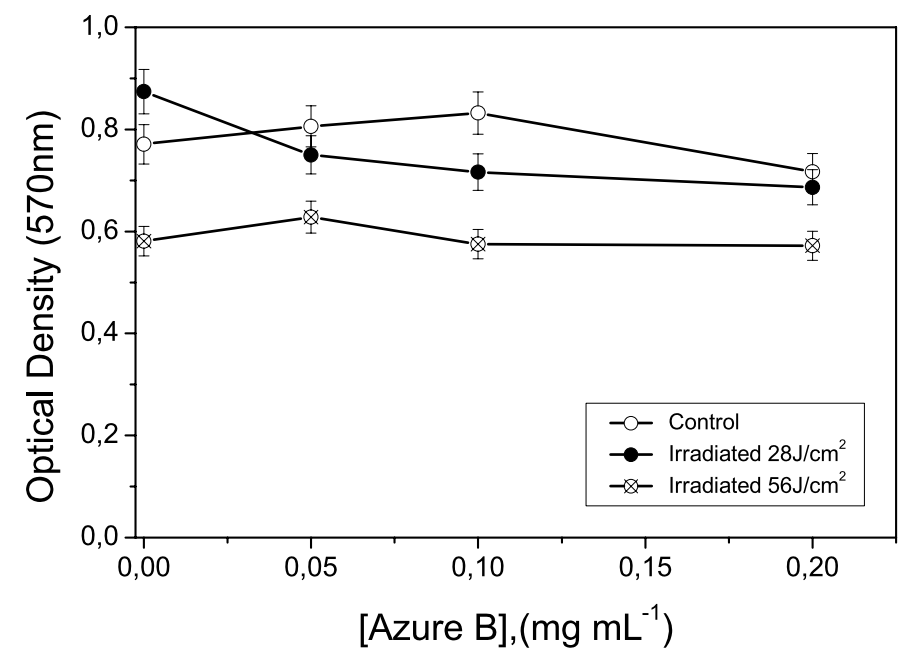

Fig. 2. Azure B effect before (०) and after the diode laser irradiation with DE of $28 \mathrm{~J} / \mathrm{cm}^{2}(\bullet)$ and $56 \mathrm{~J} / \mathrm{cm}^{2}(\otimes)$. Candida albicans suspensions $\left((1-5) \times 10^{5}\right.$ cells ml $\left.^{-1}\right)$ were irradiated with diode laser $(684 \mathrm{~nm})$.

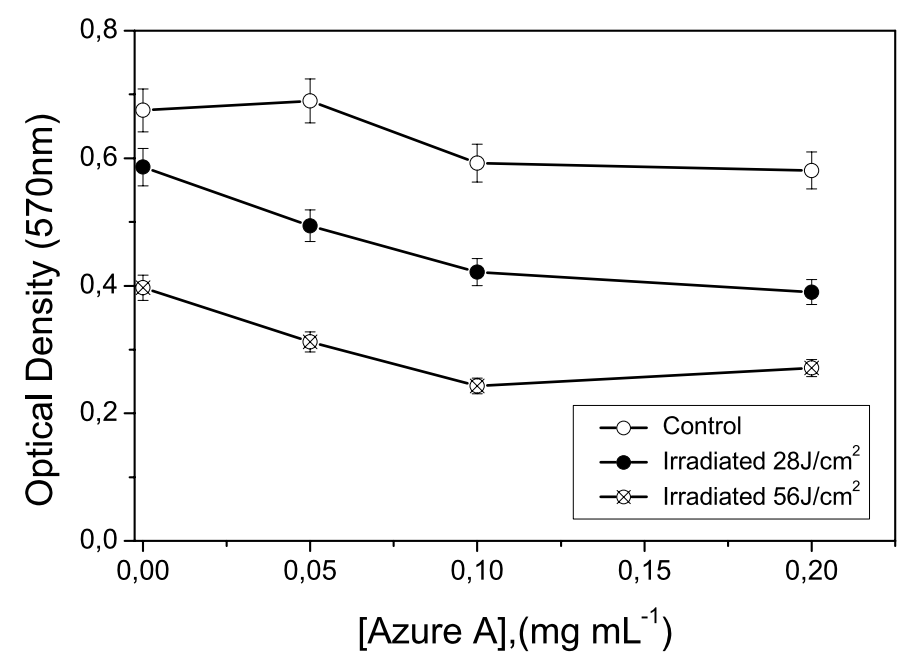

Fig. 3. Azure A effect before (०) and after the diode laser irradiation with DE of $28 \mathrm{~J} / \mathrm{cm}^{2}(\bullet)$ and $56 \mathrm{~J} / \mathrm{cm}^{2}(\otimes)$. Candida albicans suspensions $\left((1-5) \times 10^{5}\right.$ cells ml $\left.^{-1}\right)$ were irradiated with diode laser $(684 \mathrm{~nm})$.

Figure 3 demonstrates the data related to the Azure A effect on C. albicans cultures. Differently of the Azure B behavior, it is detected an effective action of Azure A, when submitted to the radiation. In fact, Azure A reduced the growth of C. albicans yeasts in approximately $30 \%$ after an irradiation $28 \mathrm{~J} / \mathrm{cm}^{2}$ and near 50-60\% subsequently to the irradiation $56 \mathrm{~J} / \mathrm{cm}^{2}$.

Figure 4 presents the behavior of Azure A thiocyanate. Interestingly, this compound demonstrates an intermediary action, when compared with Azures A and B, since an irradiation $28 \mathrm{~J} / \mathrm{cm}^{2}$ does not precludes the growth of $C$. albicans, which only occurs with $56 \mathrm{~J} / \mathrm{cm}^{2}$.

Similarly to Azure B, Azure B $\mathrm{TF}_{4}$ does not inhibit the growth of the yeasts with both radiation conditions evaluated in the present work, in agreement with the respective plot (Fig. 5). 


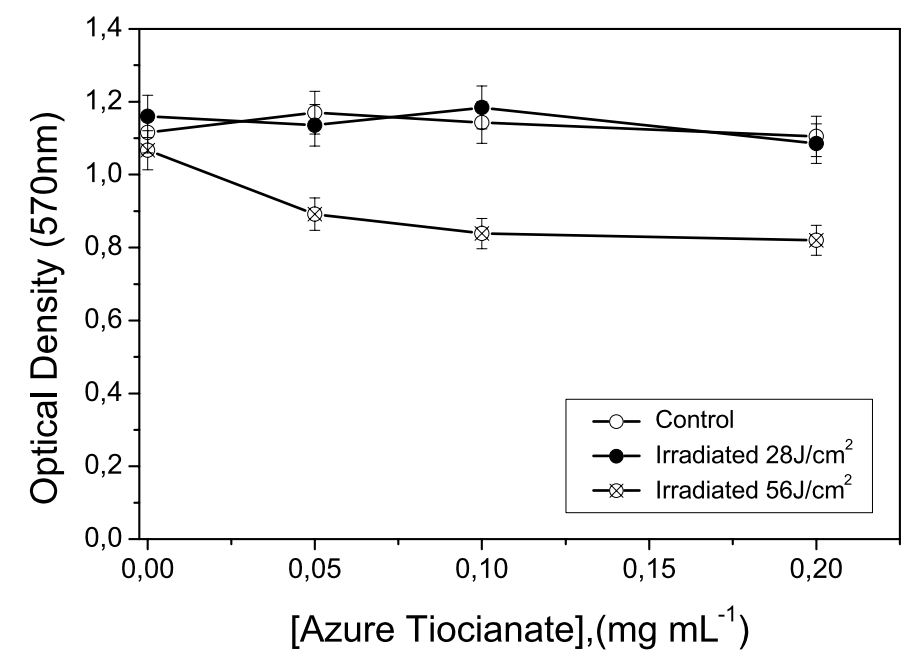

Fig. 4. Azure A thiocyanate effect before (०) and after the diode laser irradiation with DE of $28 \mathrm{~J} / \mathrm{cm}^{2}(\bullet)$ and $56 \mathrm{~J} / \mathrm{cm}^{2}(\otimes)$.

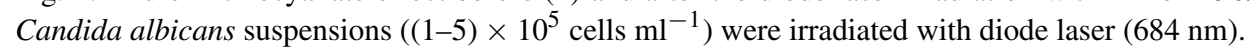

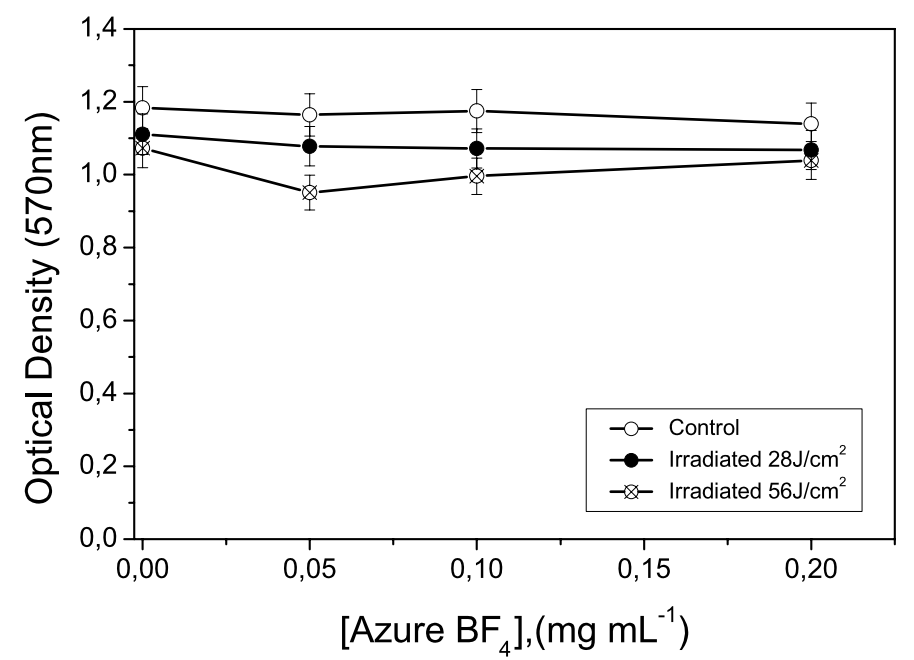

Fig. 5. Azure B tetrafluoroborate effect before (०) and after the diode laser irradiation with DE of $28 \mathrm{~J} / \mathrm{cm}^{2}(\bullet)$ and $56 \mathrm{~J} / \mathrm{cm}^{2}$

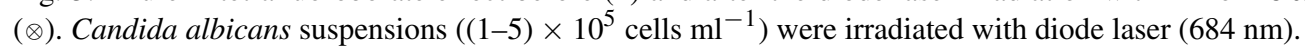

On the other hand, the Azure A eosinate behavior, presented in Fig. 6, is very close to that obtained with Azure A, being characterized by $20 \%$ of growth inhibition with $28 \mathrm{~J} / \mathrm{cm}^{2}$ and around $50-60 \%$ with $56 \mathrm{~J} / \mathrm{cm}^{2}$.

The present results demonstrate that the photodynamic action of distinct types of Azure (Azure A, Azure $\mathrm{B}$, Azure A thiocyanate, Azure $\mathrm{B} \mathrm{BF}_{4}$ and Azure A eosinate) compounds is quite different regarding the inhibition of $C$. albicans growth. Indeed, some phenothiazinium derivatives, such as Azure B and Azure $\mathrm{B} \mathrm{BF}_{4}$, are not effective to inhibit $C$. albicans growth under both conditions of irradiation analyzed in this work. However, Azure A and Azure A eosinate presented auspicious results when irradiated with $56 \mathrm{~J} / \mathrm{cm}^{2}$. These Azure compounds have significant potential to be employed as PS in PDT, especially 


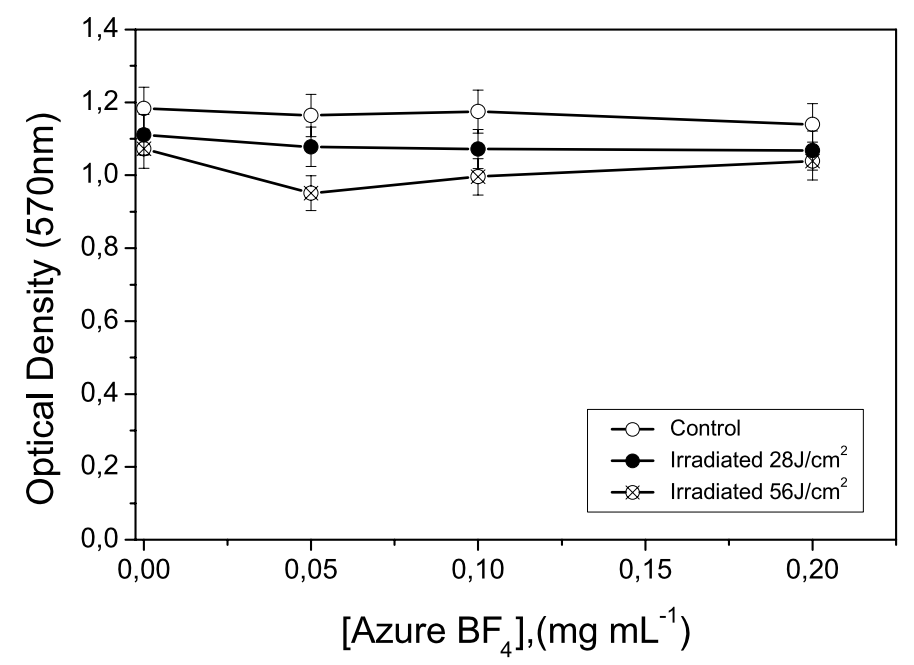

Fig. 6. Azure A eosinate effect before (०) and after the diode laser irradiation with DE of $28 \mathrm{~J} / \mathrm{cm}^{2}(\bullet)$ and $56 \mathrm{~J} / \mathrm{cm}^{2}(\otimes)$. Candida albicans suspensions $\left((1-5) \times 10^{5}\right.$ cells ml $\left.^{-1}\right)$ were irradiated with diode laser $(684 \mathrm{~nm})$.

in cases of superficial Candida infection, such as oral and vaginal candidosis, as well as nail and skin infections. In fact, it is well known that the accessibility to the biological region affected is a fundamental pre-requisite to the effectiveness of PDT. This occurs because the local concentration of excited species (photosensitizer) depends directly on the quantum yield and this phenomenon is consequence of the light intensity in contact with the photosensitizer.

The phenothiazinium dyes have simple tricyclic planar structures, typically cationic in nature. The most widely used compounds are methylene blue (MB) and toluidine blue $\mathrm{O}$ (TBO). Both are efficient producers of singlet oxygen and the maximum absorption wavelength in water is $656 \mathrm{~nm}$ for MB and $625 \mathrm{~nm}$ for TBO, respectively [7].

According to Wainwright et al. [21], cationic photosensitizers are more efficient than their neutral or anionic compounds in the photodynamic antimicrobial therapy. This information could be associated to the fact that Azure A presents effective action against $C$. albicans in opposite to the Azure B behavior. In fact, the molecular structure of Azure B presents a methyl radical as substituent group of the hydrogen found in the position called "R3" in Azure A. Considering that this compound present a cationic charge, the higher electronic density of Azure B could, through inductive effect, to decrease the intensity of this positive charge. This fact is plausible due to the higher electronic density of the methyl group when compared with the hydrogen. fungi present much more complex targets than bacteria. Yeasts possess a thick external wall composed of a mixture of glucan, mannan, chitin and lipoproteins and separated from the plasma membrane by a periplasmic space. Uptake of exogenous substances by fungi is generally adversely affected by lipophilicity and positively affected by hydrophilicity and the presence of charged groups [7].

In this context, it is relevant to note that the higher polarity of Azure A when compared with Azure B must to favor the occurrence of higher number of hydrogen bonds with the chemical neighborhood in aqueous medium. Thus, two important effects can be inferred of this significant physical-chemical difference. The first one is the lower tendency of aggregation of Azure A in comparison with Azure B, which is a well-known phenomenon associated to a decrease of the photodynamic efficacy. The second 
one would be the higher tendency of Azure A to interact with the external membranes of Fungi due to its higher polarity, which must to facilitate the interaction with the polar groups of the Candida membranes.

Donnelly et al. [7] inferred that there should be no difference in susceptibility to PACT between organisms resistant or susceptible to conventional antifungals. This occurs because the oxidation caused by the photosensitizer is non-specific and there are no cellular defenses against it. Besides, it is unlikely that fungi could readily evolve resistance to singlet oxygen and mutagenesis has never been associated to PDT.

C. albicans, as well as other yeasts are more resistant to PACT than gram-positive bacterial cells, necessitating higher drug and light doses [26]. This is probably due to the presence of a nuclear membrane, to the greater cell size and the reduced number of targets for singlet oxygen per unit volume of cell [26].

Several studies have reported the effectiveness of PACT against Candida species [3,5,7]. However, they are considerably less susceptible to photodynamic killing than several bacteria [25]. In fact, doses of TBO as high as $2.0 \mathrm{mg} \cdot \mathrm{ml}^{-1}$ have been required to induce high levels of inhibition (499\%).

In conclusion, it is possible to infer that it would be plausible to obtain a higher inhibition of C. albicans growth with Azure A and Azure A eosinate employing a more intense source of light or a higher concentration of these phenothiazinium derivatives. This new trials are being developed and soon will be published.

\section{Conclusions}

The present article evaluates a series of Azures compounds in order to identify new prototypes of photosensitizer (PS). The results demonstrated that the different phenothiazinium derivatives have quite distinct inhibitory behaviors regarding the $C$. albicans growth. The more effective results were obtained with Azure A and Azure A eosinate. On the other hand, it is clear that some phenothiazinium compounds, such as Azure $\mathrm{B}$ and Azure $\mathrm{B} \mathrm{BF}_{4}$, do not present real possibilities of application as photosensitizers. In fact, the higher polarity of the molecular structure of Azure A when compared with Azure B must be a decisive factor to propitiate this more effective photodynamic result. In this way, new tests are necessary varying concentration and light intensity to obtain a more complete analysis of the potential as photosensitizer of Azure A and Azure A eosinate, which were the more effective compounds analyzed in this manuscript. Furthermore, it is important to notice that the spectroscopic measurements of optical density was quite capable to determine the growth inhibition of $C$. albicans, and, in this way, can be considered an interesting alternative in comparison with conventional methodologies in order to develop microbiological analyzes.

\section{Acknowledgements}

The authors thank to FAPESP for financial grant (J.P. Lyon, 2007/07162-5; M.R. Rodrigues, 2002/00272-6; H.P.M. de Oliveira, 06/56701-3; L. Codognoto, 08/50588-6) and CNPq (H.P.M. de Oliveira, 479655/2008-1). Thanks to A. Lima for assistance in the laboratory.

\section{References}

[1] A. Asilian and M. Davami, Comparison between the efficacy of photodynamic therapy and topical paromomycin in the treatment of Old World cutaneous leishmaniasis: a placebo-controlled, randomized clinical trial, Clin. Exp. Dermatol. 31 (2006), 634-637. 
[2] M. Bhatti, A.M. Robert, S. Meghji, B. Henderson and M. Wilson, Effect of dosimetric and physiological factors on the lethal photosensitization of Porphyromonas gingivalis in vitro, Photochem. Photobiol. 65 (1997), 1026-1031.

[3] J.M. Bliss, C.E. Bigelow, T.H. Foster and C.G. Haidaris, Susceptibility of Candida species to photodynamic effects of photofrins, Antimicrob. Agents Chemother. 48 (2004), 2000-2006.

[4] W.C. Bristo, R. Hudson, T.A. Paget and R.W. Boyle, Potential of cationic porphyrins for photodynamic treatment of cutaneous leishmaniasis, Photodiag. Photodyn. Ther. 3 (2006), 162-167.

[5] S.C. de Souza, J.C. Junqueira, I. Balducci and C.Y. Koga-Ito, Photosensitisation of different Candida species by low power laser light, J. Photochem. Photobiol. B 83 (2006), 34-38.

[6] J. Dobson and M. Wilson, Sensitization of oral bacteria in biofilms to killing by light from a low-power laser, Arch. Oral Biol. 37 (1992), 883-887.

[7] R.F. Donnelly, P.A. McCarron, M.M. Tunney and A.D. Woolfson, Potential of photodynamic therapy in treatment of fungal infections of the mouth. Design and characterization of a mucoadhesive patch containing toluidine blue O, J. Photochem. Photobiol. B 86 (2007), 59-69.

[8] A.S. Garcez, F.R. Souza, S.C. Núñez, J.M. Kather and M.S. Ribeiro, Terapia fotodinâmica em odontologia - laser de baixa potência para redução microbiana, Rev. APCD 57 (2003), 223-226.

[9] L. Gonçalves, Efeito de fotoativadores utilizados na irradiação laser intracanal, MSc dissertation (Mestrado em Odontologia), Universidade de São Paulo, 2005.

[10] J.P. Lyon, S.C. Costa, V.M.G. Totti, M.F.V. Munhoz and M.A. Resende, Predisposing conditions for Candida spp. carriage in the oral cavity of denture wearers and individuals with natural teeth, Can. J. Microbiol. 52 (2006), 462-467.

[11] L.M. Moreira, F.V. Santos, J.P. Lyon, M.M. Costa, C.P. Soares and N.S. Silva, Photodynamic therapy (PDT): porphyrins and phthalocyanines as photosensitizers (PS), Aust. J. Chem. 61 (2008), 741-754.

[12] B. Narayana, T. Cherian and A. Facile, Spectrophotometric method for the determination of periodate using Azure B, J. Braz. Chem. Soc. 1 (2005), 978-981.

[13] P.M. Pinto, R.C.B.W. Oliveira, J.P. Lyon, V.F. Cury, R.R. Arantes, C.Y. Koga-Ito and M.A. Resende, In vitro antifungal susceptibility of clinical isolates of Candida spp. obtained from patients with different predisposing factors to candidosis, Microbiol. Res. 163 (2008), 579-585.

[14] M.A. Resende, L.V.N. Franco, R.C.B.W. Oliveira, C.Y. Koga-Ito and J.P. Lyon, Prevalence and antifungal susceptibility of yeasts obtained from the oral cavity of elderly individuals, Mycopathologia 162 (2006), 39-44.

[15] C. Romagnoli, D. Mares, G. Sacchetti and A. Bruni, The photodynamic effect of 5-(4-hydroxy-1-butinyl)-2,2-biethienyl on dermatomycetes, Mycol. Res. 102 (1998), 1519-1524.

[16] P. Saballs, J.M.T. Rodrigues and M. Salvadó, La candidemia en el síndrome de inmunodeficiencia adquirida. Estudio retrospectivo de nueve casos, Rev. Iberoam. Micol. 17 (2000), 2-5.

[17] D.J. Sheehan, C.A. Hitchcock and C.M. Sibley, Current and emerging azole antifungal agents, Clin. Microbiol. Rev. 12 (1999), 40-79.

[18] N.S. Silva, C.M. Ribeiro, A.H.A. Machado and C. Pacheco-Soares, Ultrastructural changes in Tritrichomonas foetus after treatments with $\mathrm{AlPcS}_{4}$ and photodynamic therapy, Vet. Parasitol. 146 (2007), 175-181.

[19] S.C. Souza, J.C. Junqueira, I. Balducci, C.Y. Koga-Ito, E. Munin and A.O.C. Jorge, Photosensitization of different Candida species by low power laser light, J. Photochem. Photobiol. B 12 (2006), 34-38.

[20] S. Taniguchi, N. Suzuki, M. Masuda, S. Hisanaga, T. Iwatsubo, M. Goedert and M. Hasegawa, Inhibition of heparininduced tau filament formation by phenothiazines, polyphenols, and porphyrins, J. Biol. Chem. 280 (2005), 7614-7623.

[21] M. Wainwright, Photodynamic antimicrobial chemotherapy, J. Antimicrob. Chemother. 42 (1998), 13-19.

[22] M. Wainwright, Phenotiazinium photosensitizers: V. Photobactericidal activities of chromophore-methylated phenotiazinium salts, Dyes Pigm. 73 (2007), 7-12.

[23] M. Wainwright and R.M. Giddens, Phenotiazinium photosensitizers: choices in synthesis and applications, Dyes Pigm. 57 (2003), 245-257.

[24] I.C.J. Zanin, A. Brugnera Júnior and R.B. Gonçalves, Susceptibility of Streptococcus mutans biofilms to photodynamic therapy: an in vitro study, J. Antimicrob. Chemother. 56 (2005), 324-330.

[25] B. Zeina, J. Greenman, D. Corry and W.M. Purcell, Cytotoxic effects of antimicrobial photodynamic therapy on keratinocytes in vitro, Braz. J. Dermatol. 146 (2002), 568-573.

[26] B. Zeina, J. Greenman, W.M. Purcell and B. Das, Killing of cutaneous microbial species by photodynamic therapy, Braz. J. Dermatol. 144 (2001), 274-278. 


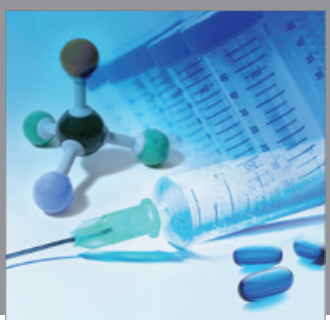

International Journal of

Medicinal Chemistry

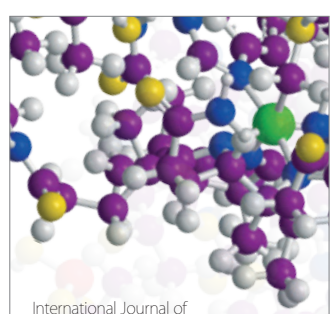

Carbohydrate Chemistry

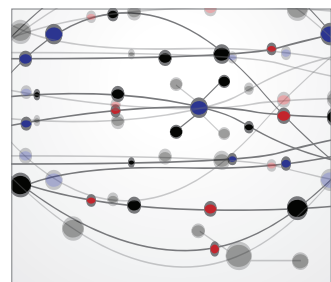

The Scientific World Journal
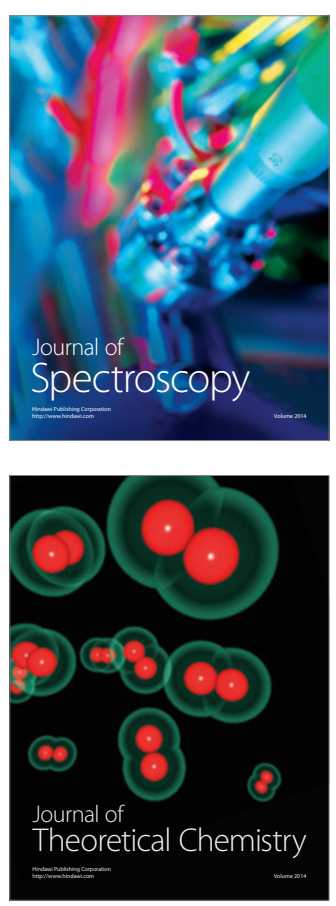
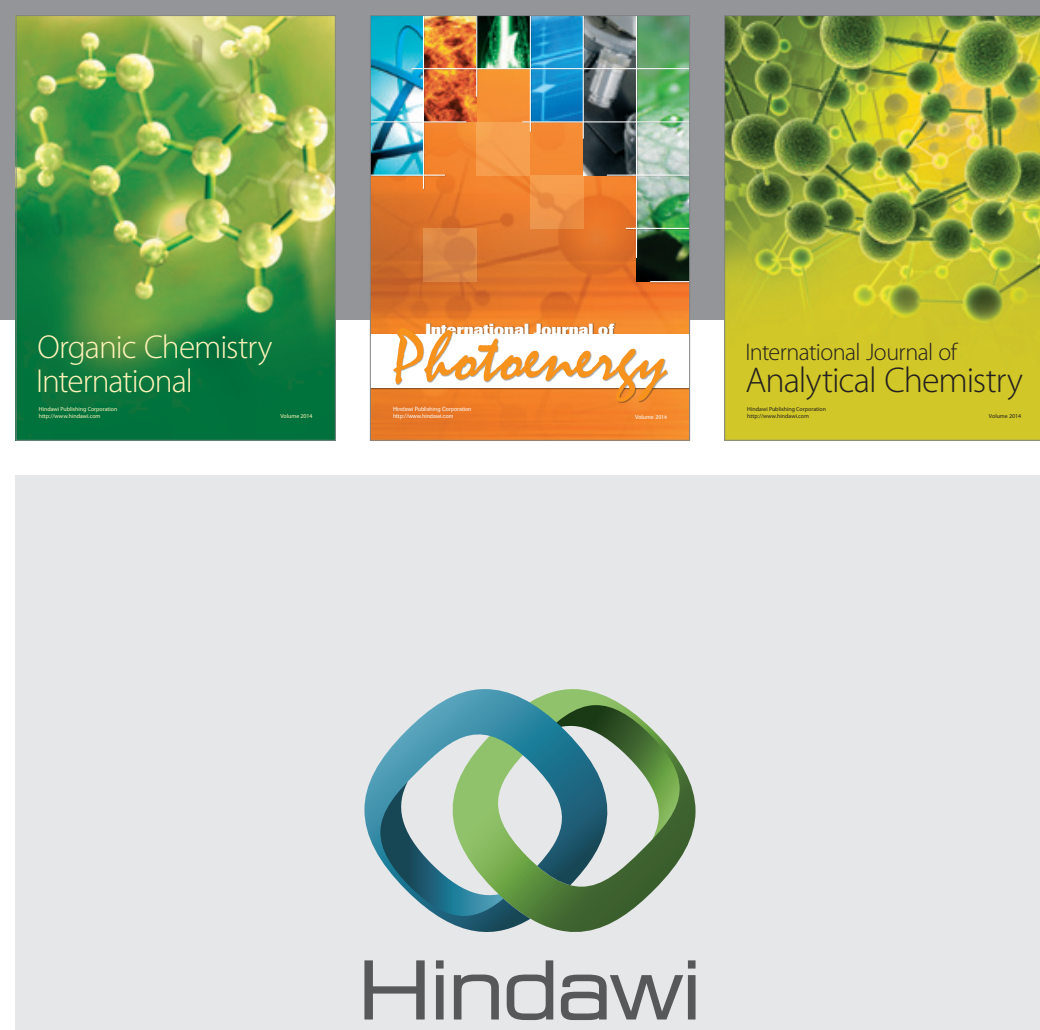

Submit your manuscripts at

http://www.hindawi.com
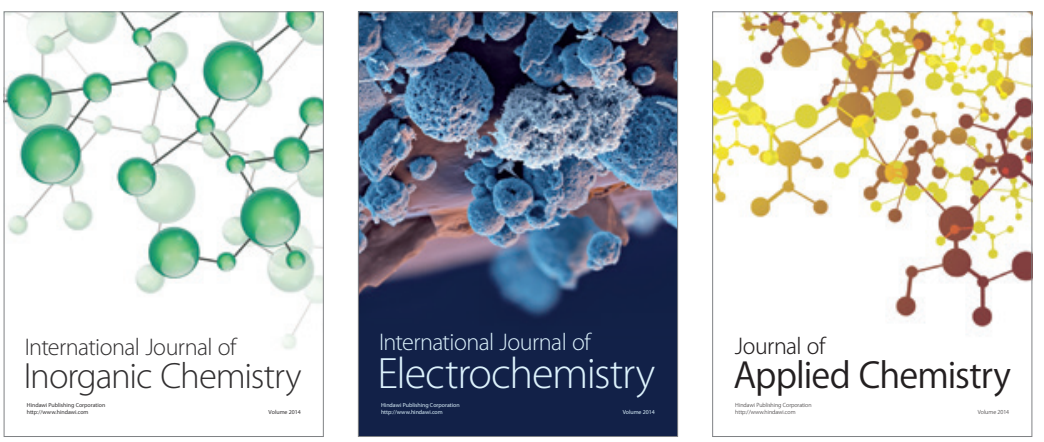

Journal of

Applied Chemistry
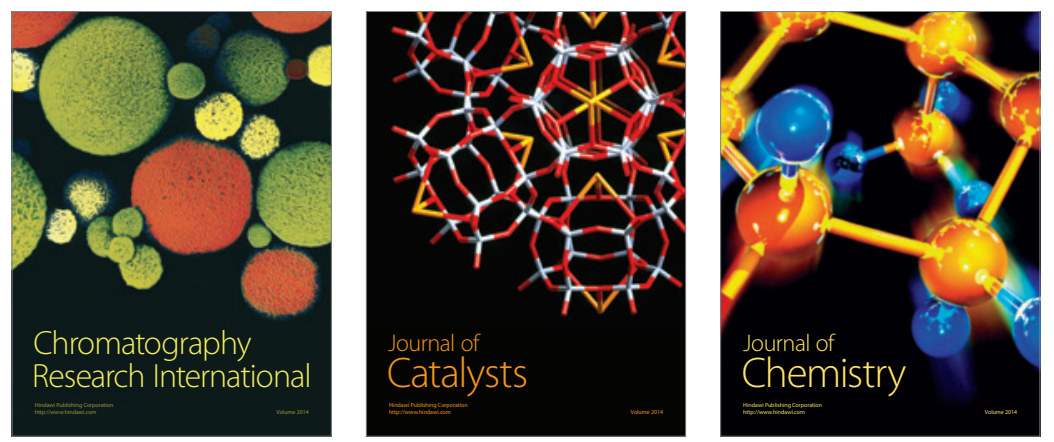
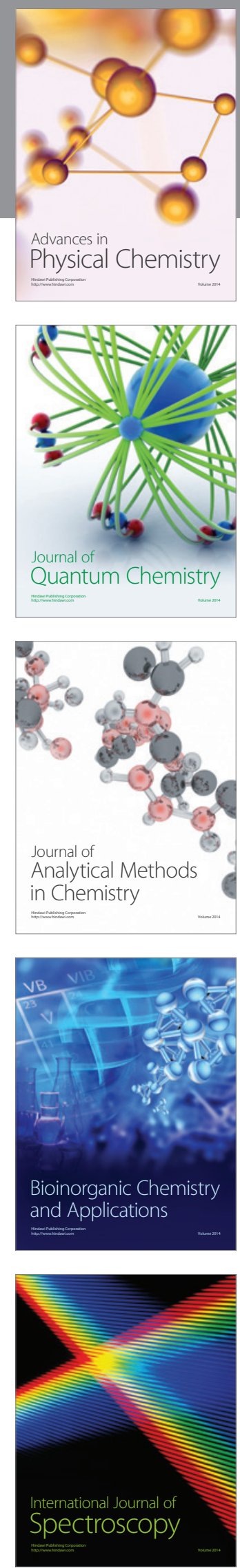